\title{
Il ciclo cellulare: cos'è, come è stato e come sarà studiato
}

\author{
Sara Nardi ${ }^{1}$, Duccio Lombardi ${ }^{2}$ \\ ${ }^{1}$ Unità di Nefrologia, Ospedale Universitario Pediatrico Meyer, Firenze \\ ${ }^{2}$ Dottorando in Area del Farmaco e Trattamenti Innovativi, Dipartimento di Medicina Sperimentale e Clinica, Centro d'Eccellenza DENOTHE, \\ Università degli Studi di Firenze, Firenze
}

\begin{abstract}
The cell cycle: what it is, how it has been studied and how it will be studied
The cell cycle has a pivotal role in many aspects of cell's life. The metabolic state of the cell and its decision to proliferate or even to die, as well as physiological or pathological conditions, are some of the aspects deeply influenced by the cell cycle and that, in their turn, influence it. The molecular basis of this extremely complex process has been extensively studied, nevertheless a lot of information is still missing. Indeed, old widely applied techniques as thymidine analogues incorporation have been shown to be inadequate in many current experimental settings or if employed with new scientific methodologies. Conversely, new tools recently developed, as the FUCCI technology, are a great promise and will enable to deepen our comprehension of the cell cycle by allowing scientists to study it in vivo through live imaging. With this contribution, the basis of the cell cycle and of its regulation as well as old and new techniques to study it, will be revised.
\end{abstract}

Keywords: Cell cycle, Cyclins, Cyclin dependent kinases, FUCCI technology

\section{Il ciclo cellulare}

Il ciclo cellulare è quel processo attraverso cui una cellula si moltiplica provvedendo, così, alla generazione di nuove cellule figlie. Esso è perciò fondamentale nei processi di sviluppo e crescita di un organismo, è finalizzato alla regolazione del turnover e dell'omeostasi di un tessuto ed entra in gioco anche per la sostituzione di cellule perse in seguito a un danno.

Nelle cellule eucariotiche, il ciclo cellulare si divide in una prima fase, detta interfase, costituita in maniera sequenziale dalle fasi G1, Se G2, e in una fase di mitosi, o M, in cui avviene la divisione fisica tra la cellula madre e quella che sarà chiamata cellula figlia (1). Nell'interfase, la cellula cresce di volume ed è particolarmente attiva nelle sue funzioni metaboliche, come l'ossidazione del glucosio, e genomiche, ossia trascrizione, traduzione e replicazione del DNA. La fase G1 (o Gap 1), è una fase preparatoria in cui la cellula aumenta di dimensioni e sintetizza numerose molecole necessarie nelle successive fasi del ciclo cellulare, soprattutto per quanto ri-

Accepted: May 21, 2015

Published online: June 15, 2015

Indirizzo per la corrispondenza:

Dr.ssa Sara Nardi

Centro DENOTHE

Viale Pieraccini 6

50139 Firenze

sara.nardi05@gmail.com guarda la fase $S$, momento in cui il materiale genomico sarà duplicato per dare due copie identiche che saranno ripartite con le cellule figlie (1). Affinché il ciclo cellulare abbia un buon esito è, però, fondamentale che la duplicazione del DNA avvenga una sola volta per ciclo. Per garantire ciò, in fase G1 tardiva, alcune proteine iniziatrici, tra cui Cdt1 e Cdc6, si legano ai siti da cui parte la duplicazione del DNA (origini di replicazione), affinché solo questi siano funzionali e attivi durante la fase $\mathrm{S}$ e affinché lo stesso locus genetico sia duplicato una sola volta (1). Al termine della fase $\mathrm{S}$, quando il genoma della cellula sarà stato duplicato, le proteine che costituiscono le origini di replicazione saranno disassemblate: $\mathrm{Cdc} 6$ viene inattivata da una proteina chinasi e degradata, mentre Cdt1 viene, a sua volta, inibita e degradata durante la fase M. Terminata la duplicazione del DNA, la cellula è pronta a entrare nella fase successiva, la fase G2 (o Gap 2). In questa fase la cellula inizia a formare il fuso mitotico, che servirà poi nella fase $\mathrm{M}$ per separare i cromosomi, mentre il DNA si condensa al fine di rendere più semplice una bilanciata segregazione del materiale genomico. Inoltre l'attivazione di altre proteine favorisce la degradazione dell'involucro nucleare, consentendo così il passaggio alla successiva fase $M$. Durante la mitosi si verificano, infine, la separazione cromosomica e la citodieresi, che porta alla separazione fisica tra cellula madre e cellula figlia.

La durata del ciclo cellulare può variare molto a seconda del tessuto o del contesto fisiopatologico in cui questo si trova. Anche la durata delle singole fasi non è la stessa per tutte le cellule: in una cellula adulta la fase G1 è normal- 
mente la fase più lunga, mentre le fasi G2 e M sono abbastanza brevi. Al contrario, in una cellula embrionale le fasi G1 e $\mathrm{G} 2$ sono estremamente rapide, facendo sì che si abbia un elevato tasso di duplicazione cellulare.

\section{Regolazione della progressione attraverso il ciclo cellulare: cicline e Cdk}

La transizione tra le diverse fasi e la progressione nel ciclo cellulare sono regolate dall'azione combinata di due classi di proteine, le cicline e le chinasi ciclina-dipendenti (Cyclin-dependent kinases, Cdk), nonché dal susseguirsi di attivazione e inattivazione dei complessi che queste due formano. Le cicline, scoperte inizialmente durante studi sugli invertebrati marini (2), sono suddivise in quattro tipi, sono fase-specifiche e, associandosi alle Cdk, ne determinano l'attivazione e la specificità d'azione. Le Cdk sono, invece, proteine serina-treonina chinasi dotate di una subunità che riconosce e lega la ciclina e di una subunità funzionale che riconosce e attiva/disattiva varie proteine bersaglio coinvolte nella duplicazione cellulare. Esistono quattro tipi di cicline e almeno sei diverse Cdk. A differenza delle cicline, che vengono sintetizzate per via trascrizionale e inattivate tramite una degradazione controllata, le Cdk sono sempre espresse, ma in forma inattiva, e si attivano solo in seguito al legame con la specifica ciclina.

I complessi ciclina-Cdk sono regolati a vari livelli. In primis il complesso tra le due proteine si ha solo qualora la specifica ciclina sia espressa dalla cellula; in secondo luogo, il complesso ciclina-Cdk è attivato o inibito da altre proteine regolatorie del ciclo cellulare, come la chinasi attivatoria Cak, la chinasi inibitoria Wee1 e la fosfatasi attivatoria Cdc25. Tali proteine regolatorie sono attivate durante i punti di controllo del ciclo cellulare e inattivano temporaneamente il complesso ciclina-Cdk per dare modo alla cellula di riparare a eventuali errori o di completare step che devono avvenire in una data fase: solo così la cellula potrà procedere nel ciclo cellulare. Infine, quando la cellula deve passare alla fase successiva e il complesso ciclina-Cdk ha esaurito la sua funzione, la ciclina viene degradata e si attiva il complesso della fase successiva.

Per la loro necessità di rispondere a una grande varietà di stimoli, le cellule eucariotiche hanno sviluppato varie Cdk che, di volta in volta, si associano a una specifica ciclina (3). In ogni periodo del ciclo cellulare, sarà, pertanto, attivo un solo tipo di complesso Cdk-ciclina e, a seconda del complesso attivo, saranno fosforilate diverse molecole bersaglio, quali proteine necessarie per l'assemblaggio del fuso mitotico o del nucleo cellulare o varie proteine citoscheletriche implicate nei cambiamenti conformazionali che la cellula deve compiere per dividersi.

Altri due importanti complessi enzimatici che contribuiscono a regolare l'alternanza delle fasi del ciclo cellulare sono il complesso che promuove l'anafase (Anaphase Pro- moting Complex, APC) e il complesso SCF (complesso multiproteico formato da Skp1, cullina e F-box). Questi due complessi riconoscono proteine che devono essere degradate e, insieme all'azione di altri enzimi, le dirigono verso il proteasoma per la degradazione finale, permettendo il passaggio alla fase successiva (4). Mentre SCF agisce principalmente al termine della fase $\mathrm{G} 1$, il complesso APC è attivo nella parte finale della fase $M$, ove rappresenta il principale punto di controllo, e dove regola la degradazione delle cicline della fase $M$ e della Geminina e attiva la sintesi delle proteine per la successiva fase $\mathrm{G} 1$.

\section{Controllo del ciclo cellulare}

In un contesto multicellulare, il ciclo cellulare è regolato sia da segnali intrinseci $(5,6)$, che consentono il passaggio alla fase successiva del ciclo solo dopo aver verificato il corretto completamento della fase in corso, che da segnali estrinseci, come la risposta a segnali provenienti dall'ambiente extracellulare (5). Per il ruolo strategico e l'importanza che il ciclo cellulare riveste per la sopravvivenza della cellula, il controllo tra i passaggi di fase è finemente regolato a livello di tre punti di controllo, o checkpoint, fondamentali per la progressione nel ciclo cellulare. Se, infatti, la cellula non avesse completato gli step necessari o non fosse comunque in possesso dei requisiti richiesti per il superamento della fase in corso, un segnale di stop ritarderebbe l'ingresso nella fase successiva, bloccando la cellula temporaneamente presso tali checkpoint. Durante il ritardo concesso dal sistema di controllo, la cellula completa tutti i cambiamenti necessari per passare alla fase successiva e solo allora il ciclo potrà riprendere normalmente. II primo dei punti in cui opera il sistema di controllo è localizzato alla fine della fase G1: in presenza di un ambiente intra ed extracellulare favorevole il superamento di questo checkpoint permette alla cellula di entrare in fase $S$ e di duplicare il proprio materiale genetico. II secondo punto di controllo è posto alla fine della fase G2: un ambiente favorevole e la corretta replicazione del DNA consentono il passaggio da qui alla fase M. Infine, un terzo punto di controllo si trova tra le sotto-fasi mitotiche metafase e anafase: esso verifica che i cromosomi siano correttamente allineati e che siano connessi al fuso mitotico, così da dare il segnale che la cellula può completare la mitosi e la citodieresi.

La capacità di intraprendere il ciclo cellulare dipende, però, anche dai segnali esterni che la cellula riceve. Tutte le cellule sono teoricamente in grado di effettuare il ciclo cellulare, ma non tutte lo fanno: ci sono, infatti, cellule normalmente ciclanti, dette labili, che ricevono continuamente segnali mitogeni, come le cellule embrionali, le cellule degli epiteli di rivestimento e i tessuti ematopoietici. Le cellule che si trovano, invece, in fase di quiescenza si definiscono stabili: queste cellule non si dividono, ma, se ricevono uno stimolo mitogeno, sono ancora in grado di intraprendere il 
ciclo cellulare. Ne sono un esempio le cellule del parenchima dei tessuti ghiandolari, i fibroblasti e le cellule endoteliali. Infine, ci sono cellule perenni che, una volta differenziate, non proliferano o per mancanza di segnali che ne inducano la proliferazione o perché la duplicazione è impedita per motivi fisiologici. Questo è il caso dei podociti, cellule altamente specializzate con un corpo voluminoso che, attraverso la formazione dei processi pedicellari, sono responsabili della corretta filtrazione glomerulare. I podociti non possono entrare nel ciclo cellulare in quanto ciò comporterebbe il cambiamento della loro caratteristica forma e la perdita di adesione dei processi pedicellari, e quindi del podocita stesso dai capillari glomerulari, evento che sarebbe immediatamente seguito dall'impossibilità di filtrazione con conseguente danno renale (7).

\section{Tecniche per lo studio del ciclo cellulare}

La regolazione della proliferazione cellulare è centrale per la morfogenesi dei tessuti durante lo sviluppo degli organismi multicellulari. Inoltre, la perdita di controllo della proliferazione cellulare è alla base di molte patologie, tra cui ovviamente spicca quella neoplastica. È, quindi, di particolare importanza poter identificare le cellule che stanno replicando o che hanno già replicato il loro DNA all'interno di una popolazione più ampia. Ciò può essere facilmente eseguito mediante citometria a flusso su cellule marcate con ioduro di propidio, molecola fluorescente che si intercala nel DNA. Analogamente, la sintesi attiva del DNA può essere quantificata allestendo una coltura di cellule in presenza di timidina radioattiva e misurando l'incorporazione di radioattività che si ha in una data unità di tempo (8). La stessa procedura può essere però condotta anche impiegando analoghi della timina come la bromodeossiuridina $(\operatorname{BrdU})(9,10)$. In seguito all'incubazione con BrdU, le cellule in fase $S$ incorporano la molecola nel materiale acidonucleico al posto della timidina. Tali cellule sono in seguito identificate utilizzando un anticorpo anti-BrdU, e ciò rende così possibile valutare la percentuale di cellule nella fase $\mathrm{S}$ mediante microscopia a fluorescenza o citofluorimetria. Per avere, però, un'idea più precisa dell'esatta fase del ciclo in cui si trova una cellula, è possibile fare un'analisi multiparametrica combinando le informazioni derivate dalla colorazione con BrdU con quelle ricavate marcando con ioduro di propidio. Così facendo, sarà possibile distinguere nettamente le cellule in G1 da cellule in fase $S$ precoce, o cellule in fase $S$ tardiva da cellule in G2/M. Questo ultimo approccio è molto utilizzato per studiare gli effetti di diversi stimoli e agenti farmacologici sulla regolazione della progressione attraverso le diverse fasi del ciclo cellulare.

Altra molecola ampiamente utilizzata per l'analisi della proliferazione cellulare è il CFSE (Carbossifluoresceina Succinimidil Estere), la quale è totalmente permeabile alla membrana cellulare e che, pertanto, riesce a garantirsi pie- no accesso all'ambiente intracellulare, dove è modificata rilasciando un segnale in fluorescenza. Questo segnale si dimezza ogni volta che la cellula si divide, in quanto equamente ripartito tra le due cellule figlie, permettendo, così, di valutare il numero di successive divisioni mediante citofluorimetria.

Analogamente a queste molecole di natura esogena, è possibile studiare il ciclo cellulare anche valutando molecole specifiche che la cellula esprime solo in dati momenti del ciclo. Quelli più impiegati sono sicuramente marker come Ki67, PCNA (antigene di proliferazione cellulare), DNA ligasi1 o la porzione C-terminale dell'elicasi-B (11-14). Tuttavia, dal momento che l'identificazione di transizioni nel ciclo cellulare richiede la rilevazione di minuscoli cambiamenti nel pattern di distribuzione e nell'intensità dei segnali di fluorescenza, questi marcatori non sono in grado di evidenziare le transizioni di fase con elevato contrasto (15).

\section{La tecnologia FUCCI}

Le tecniche citate permettono di studiare il ciclo cellulare sia in colture cellulari che in studi animali, anche se sono maggiormente impiegate negli studi in vitro, ove garantiscono una maggiore risoluzione. Tuttavia, lo studio in vitro non è in grado di mimare il comportamento fisiologico che le cellule presentano nel contesto dell'organismo in vivo. Per questo motivo sono stati sviluppati nuovi metodi di imaging che permettono di studiare il ciclo cellulare nell'animale addirittura in tempo reale, rendendo così possibile l'analisi del comportamento anche della singola cellula in vivo. Nel 2008 , un gruppo di ricercatori giapponesi ha messo a punto un nuovo strumento di transgenesi che consente di visualizzare le transizioni del ciclo cellulare di una cellula in modelli animali. Questa nuova tecnologia prende il nome di FUC$\mathrm{Cl}$ (Fluorescent Ubiquitination-based Cell Cycle Indicator) e permette di distinguere le cellule vitali che si trovano in fase G1 da quelle in fase S/G2/M mediante un sistema di imaging a due colori (15). Inizialmente, i ricercatori hanno sviluppato un modello transgenico, chiamato CAG-Fucci, in cui il fluoroforo Kusabira Orange2 (mKO2) (16) e il fluoroforo Azami Green (mAG) (17) sono stati fusi rispettivamente al Cdt1 e alla Geminina umane (15). Le due proteine chimeriche così ottenute (mKO2-hCdt1 e mAG-hGem) si accumulano nel nucleo delle cellule durante il ciclo cellulare colorando rispettivamente, in arancione, i nuclei di cellule che si trovano in fase $\mathrm{G} 1 \mathrm{e}$, in verde, i nuclei di cellule in fase S/G2/M (15). Successivamente, questo modello è stato perfezionato dallo stesso gruppo di ricerca ed è stato creato il modello Fucci2 (18). Nel Fucci2 sono stati impiegati come fluorofori la proteina monomerica mCherry (rossa) (19) e una variante monomerica della proteina mVenus (verde) (20). Questo modello transgenico è stato ulteriormente ottimizzato e reso inducibile inserendo le due sonde mVenus e mCherry sotto il controllo del locus Rosa26. Questo costrutto, o R26RFuc- 
ci2, ha il vantaggio di essere silente, se non attivato dalla somministrazione di una molecola esogena come la Doxicilina. Quando però questa viene somministrata all'animale, l'espressione della fluorescenza legata alle sonde è resa possibile e i nuclei cellulari si colorano, così, in rosso (cellule in G1) e in verde (cellule in S/G2). Durante la transizione dalla fase $\mathrm{G} 1$ alla $\mathrm{S}$, i nuclei appaiono invece gialli, permettendo così di individuare le cellule che hanno avviato la replicazione del DNA. Al contrario, le cellule in fase mitotica non appaiono colorate, in quanto la Geminina è rapidamente degradata nella fase G2 tardiva (15).

Tuttavia, i modelli appena descritti presentano problemi di inattivazione del transgene, di variabilità dei livelli di espressione dei fluorofori e necessitano di continui incroci tra l'animale ingegnerizzato con la sonda rossa e quello ingegnerizzato con la sonda verde affinché la loro progenie possa esprimere entrambe le sonde. Per superare questi ostacoli, alcuni ricercatori hanno sviluppato una variante bicistronica di Fucci, che incorpora entrambe le sonde in un singolo transgene: $m$ Venus-hGem e mCherry-hCdt1 sono state fuse tra di loro e il trascritto risultante è, perciò, in grado di produrre quantità equimolari di entrambe le sonde (21). Questa variante, o R26Fucci2aR, presenta un'espressione elevata, inducibile e ubiquitaria delle sonde e permette di studiare con un'efficacia decisamente maggiore lo stato del ciclo cellulare in linee cellulari sia in vitro che in vivo in diversi stadi della vita dell'animale (21).

La tecnologia FUCCl, in tutte le sue varianti, si è dimostrata uno strumento convincente e molto efficiente per studiare le dinamiche del ciclo cellulare con una risoluzione senza precedenti. In particolare, essa si è rivelata utile non solo per valutare la crescita e la proliferazione di una popolazione cellulare in condizioni fisiologiche o patologiche, ma ha permesso anche di analizzare l'effetto di un farmaco sul ciclo cellulare della popolazione analizzata, di studiare il profilo di ciclo cellulare di cellule tumorali e di valutare i meccanismi attraverso i quali farmaci antitumorali modulano il ciclo cellulare stesso (18).

Tuttavia, ulteriori modifiche ai sistemi già esistenti o lo sviluppo di nuove tecnologie sono costantemente richiesti per garantire strumenti sempre più affidabili e in linea con le necessità dei ricercatori. Così facendo, sarà possibile studiare nei più fini dettagli molecolari tutti i complessi aspetti del ciclo cellulare, con particolare attenzione ai compartimenti staminali identificati in molti organi, i quali potrebbero essere modulati al fine di favorire la rigenerazione d'organo.

\section{Disclosures}

Financial support: No financial support was received for this submission.

Conflict of interest: The authors have no conflict of interest.

\section{Bibliografia}

1. Harwell LH, Culotti J, Pringle JR, Reid BJ. Genetic control of the cell division cycle in yeast. Science. 1974;183(120):46-51.

2. Evans T, Rosenthal ET, Youngblom J, Distel D, Hunt T. Cyclin: a protein specified by maternal mRNA in sea urchin eggs that is destroyed at each cleavage division. Cell. 1983;33(2):389-96.

3. van den Heuvel S, Harlow E. Distinct roles for cyclin-dependent kinases in cell cycle control. Science. 1993;262(5142):2050-4.

4. Vodermaier HC. APC/C and SCF: controlling each other and the cell cycle. Curr Biol. 2004;14(18):R787-96.

5. Nurse P. A long twentieth century of the cell cycle and beyond. Cell. 2000;100(1):71-8.

6. Singh AM, Dalton S. The cell cycle and Myc intersect with mechanisms that regulate pluripotency and reprogramming. Cell Stem Cell. 2009;5(2):141-9.

7. Lasagni L, Lazzeri E, Shankland SJ, Anders HJ, Romagnani P. Podocyte mitosis - a catastrophe. Curr Mol Med. 2013;13(1): 13-23.

8. Peired A, Angelotti ML, Ronconi E, et al. Proteinuria impairs podocyte regeneration by sequestering retinoic acid. J Am Soc Nephrol. 2013;24(11):1756-68.

9. Maeshima A, Yamashita S, Nojima Y. Identification of renal progenitor-like tubularcells that participate in the regeneration processes of the kidney. J Am Soc Nephrol. 2003;14(12):3 138-46.

10. Maeshima A, Sakurai H, Nigam SK. Adult kidney tubular cell population showing phenotypic plasticity, tubulogenic capacity, and integration capability into developing kidney. J Am Soc Nephrol. 2006;17(1):188-98.

11. Leonhardt $H$, Rahn HP, Weinzierl $P$, et al. Dynamics of DNA replication factories in living cells. J Cell Biol. 2000;149(2): 271-80.

12. Essers J, Theil AF, Baldeyron C, et al. Nuclear dynamics of PCNA in DNA replication and repair. Mol Cell Biol. 2005;25(21): 9350-9.

13. Kisielewska J, Lu P, Whitaker M. GFP-PCNA as an S-phase marker in embryos during the first and subsequent cell cycles. Biol Cell. 2005;97(3):221-9.

14. Easwaran HP, Leonhardt H, Cardoso MC. Cell cycle markers for live cell analyses. Cell Cycle. 2005;4(3):453-5.

15. Sakaue-Sawano A, Kurokawa H, Morimura T, et al. Visualizing spatiotemporal dynamics of multicellular cell-cycle progression. Cell. 2008;132(3):487-98.

16. Karasawa S, Araki T, Nagai T, Mizuno H, Miyawaki A. Cyanemitting and orange-emitting fluorescent proteins as a donor/ acceptor pair for fluorescence resonance energy transfer. Biochem J. 2004;381(Pt. 1):307-12.

17. Karasawa S, Araki T, Yamamoto-Hino M, Miyawaki A. A greenemitting fluorescent protein from Galaxeidae coral and its monomeric version for use in fluorescent labeling. J Biol Chem. 2003;278(36):34167-71.

18. Sakaue-Sawano A, Kobayashi T, Ohtawa K, Miyawaki A. Druginduced cell cycle modulation leading to cell-cycle arrest, nuclear mis-segregation, or endoreplication. BMC Cell Biol. 2011;12:2.

19. Shaner NC, Campbell RE, Steinbach PA, Giepmans BN, Palmer $A E$, Tsien RY. Improved monomeric red, orange and yellow fluorescent proteins derived from Discosoma sp. red fluorescent protein. Nat Biotechnol. 2004;22(12):1567-72.

20. Nagai T, Ibata K, Park ES, Kubota M, Mikoshiba K, Miyawaki A. $A$ variant of yellow fluorescent protein with fast and efficient maturation for cell-biological applications. Nat Biotechnol. 2002;20(1):87-90.

21. Mort RL, Ford MJ, Sakaue-Sawano A, et al. Fucci2a: a bicistronic cell cycle reporter that allows Cre mediated tissue specific expression in mice. Cell Cycle. 2014;13(17):2681-96. 